\title{
Sentinel-6A precise orbit determination using a combined GPS/Galileo receiver
}

\author{
Oliver Montenbruck ${ }^{1}\left[\right.$ [D $\cdot$ Stefan Hackel $^{1}$ (]) Martin Wermuth $^{1} \cdot$ Franz Zangerl $^{2}$
}

Received: 19 June 2021 / Accepted: 16 August 2021 / Published online: 5 September 2021

(c) The Author(s) 2021

\begin{abstract}
The Sentinel-6 (or Jason-CS) altimetry mission provides a long-term extension of the Topex and Jason-1/2/3 missions for ocean surface topography monitoring. Analysis of altimeter data relies on highly-accurate knowledge of the orbital position and requires radial RMS orbit errors of less than $1.5 \mathrm{~cm}$. For precise orbit determination (POD), the Sentinel-6A spacecraft is equipped with a dual-constellation GNSS receiver. We present the results of Sentinel-6A POD solutions for the first 6 months since launch and demonstrate a 1-cm consistency of ambiguity-fixed GPS-only and Galileo-only solutions with the dual-constellation product. A similar performance (1.3 cm 3D RMS) is achieved in the comparison of kinematic and reduceddynamic orbits. While Galileo measurements exhibit 30-50\% smaller RMS errors than those of GPS, the POD benefits most from the availability of an increased number of satellites in the combined dual-frequency solution. Considering obvious uncertainties in the pre-mission calibration of the GNSS receiver antenna, an independent inflight calibration of the phase centers for GPS and Galileo signal frequencies is required. As such, Galileo observations cannot provide independent scale information and the estimated orbital height is ultimately driven by the employed forces models and knowledge of the centerof-mass location within the spacecraft. Using satellite laser ranging (SLR) from selected high-performance stations, a better than $1 \mathrm{~cm}$ RMS consistency of SLR normal points with the GNSS-based orbits is obtained, which further improves to $6 \mathrm{~mm}$ RMS when adjusting site-specific corrections to station positions and ranging biases. For the radial orbit component, a bias of less than $1 \mathrm{~mm}$ is found from the SLR analysis relative to the mean height of 13 high-performance SLR stations. Overall, the reduced-dynamic orbit determination based on GPS and Galileo tracking is considered to readily meet the altimetry-related Sentinel-6 mission needs for RMS height errors of less than $1.5 \mathrm{~cm}$.
\end{abstract}

Keywords Sentinel-6 · Jason-CS · Single-receiver ambiguity fixing · Precise orbit determination · GPS · Galileo $\cdot$ SLR · Altimetry

\section{Introduction}

Sentinel-6 is a multi-national satellite mission aiming to provide continuity of service for sea surface altimetry in the third decade of the current century (Scharroo et al. 2016; Donlon et al. 2021). It builds on the legacy of the TOPEX/Poseidon and Jason-1/-2/-3 missions, and is therefore also known as Jason Continuity of Service (CS) mission.

Oliver Montenbruck

oliver.montenbruck@dlr.de

Franz Zangerl

franz.zangerl@ruag.com

1 German Space Operations Center, Deutsches Zentrum für Luft- und Raumfahrt, 82230 Weßling, Germany

2 RUAG Space GmbH, 1120 Vienna, Austria
The prime instrument is a Poseidon-4 Ku/C-band altimeter (Cullen and Francis 2014; Donlon et al. 2021), which offers both low- and high-resolution modes and represents a major evolution over the Poseidon-3 instruments of Jason-2 and -3. Using two frequencies, ionospheric path delays in the altimeter measurements can be compensated and the total electron content below the spacecraft can be measured. The payload is complemented by the AMR-C (advanced microwave radiometer-climate quality; Maiwald et al. 2020) instrument and a GNSS radio occultation (RO) receiver (Young 2017). For precise orbit determination (POD), Sentinel-6 carries a Doppler Orbitography and Radiopositioning Integrated by Satellite (DORIS; Auriol and Tourain 2010) receiver, a redundant pair of PODRIX GPS/Galileo receivers, and a laser retro-reflector array (LRA). 


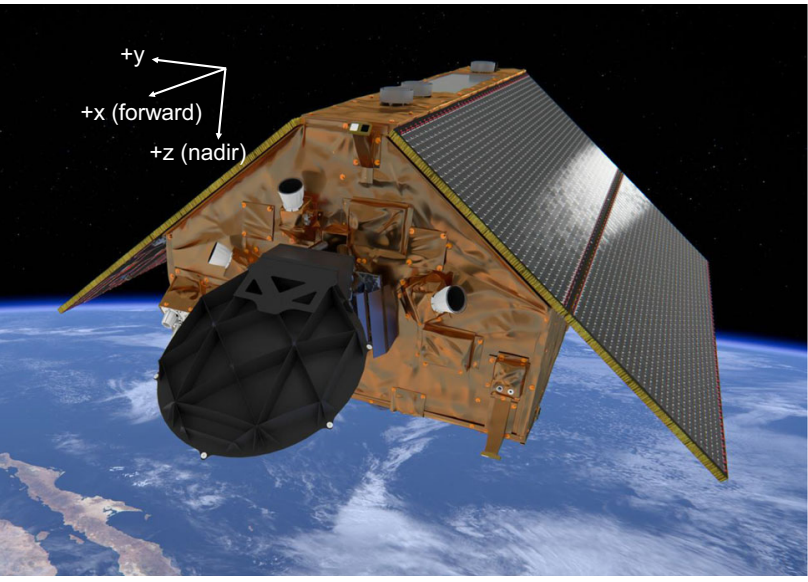

Fig. 1 Artist's drawing of the Sentinel-6A spacecraft with the dish antenna of the advanced microwave radar and three star camera baffles on the front panel. The top panel carries three GNSS choke ring antennas for the POD and RO receivers. The three axes indicate the orientation of the spacecraft body system, with $+x$ pointing in flight direction and $+z$ pointing in nadir direction during nominal operations. Image credit: ESA/ATG Medialab, CC BY-SA IGO 3.0

Sentinel-6A "Michael Freilich," the first satellite of the mission, was launched on November 21, 2020 into a low Earth orbit (LEO) of $66^{\circ}$ inclination. At a nominal altitude of $1336 \mathrm{~km}$, it completes one revolution in $112.5 \mathrm{~min}$ and its ground track repeats after 10 days (127 orbits). The spacecraft has a house-shaped body with approximate dimensions of $3.5 \mathrm{~m}$ (length), $2.4 \mathrm{~m}$ (width), and $1.8 \mathrm{~m}$ (height). Two solar panels with an individual size of about $3.6 \mathrm{~m} \times 2.4 \mathrm{~m}$ are mounted in a roof-like configuration on the portside and starboard side of the spacecraft and extend well beyond the main body (Fig. 1).

This study aims to characterize the POD accuracy achievable for Sentinel-6A with dual-constellation GNSS tracking from the PODRIX receiver. Following an overview of the receiver, calibrations of the raw observations are discussed that are required for seamless single-receiver ambiguity resolution in the POD process. Subsequently, the need for inflight calibration of the GNSS antenna is demonstrated and the resulting phase center estimates and phase patterns for GPS and Galileo frequency bands are presented. Thereafter, an overview of measurements models and dynamical models for the POD process is given with focus on the aspects of bias handling for single-receiver ambiguity fixing as well as the modeling of non-gravitational surface forces. POD results obtained for the first 6 months of the Sentinel-6A mission are presented in the following section and discussed along with an assessment of the achieved performance using various forms of self-consistency tests and external validation with satellite laser ranging (SLR) measurements.

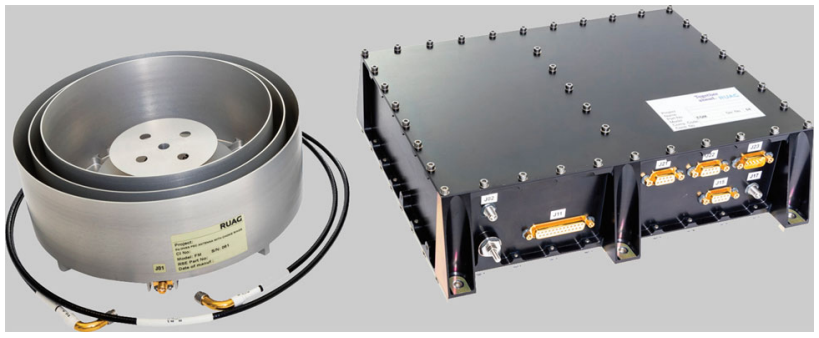

Fig. 2 PODRIX precise orbit determination receiver (right) and patch excited cup antenna with choke ring (left). Image credit: RUAG

Table 1 PODRIX observation types and corresponding RINEX (Romero 2020) identifiers

\begin{tabular}{lllll}
\hline Constellation & Code & RINEX & AGGA & Remarks \\
\hline GPS & L1 C/A & 1C & $4(2)$ & \\
& L1 P(Y) & 1W & 2 & Semi-codeless \\
& L2-CL & 2L & 4 & Long code \\
& L2 P(Y) & 2W & 2 & Semi-codeless \\
Galileo & L5-Q & 5Q & 4 & Pilot \\
& E1-C & 1C & 4 & BOC $(1,1)$ pilot \\
& E5-Q & 5Q & 4 & Pilot \\
\hline
\end{tabular}

\section{GNSS instrument and calibration}

The Sentinel-6A spacecraft is equipped with a redundant pair of triple-frequency, dual-constellation Precise Orbit Determination Receivers (PODRIX, Fig. 2) developed by RUAG, Austria. They are based on a combination of second- and fourth-generation versions of the Advanced GPS/GLONASS ASIC (AGGA), which were developed under the lead of the European Space Agency (ESA) for use in spaceborne GNSS receivers (Roselló et al. 2012).

The PODRIX combines a single AGGA-4 chip for tracking of open GPS and Galileo signals with two legacy AGGA-2 chips for GPS C/A- and semi-codeless P(Y)-code tracking. Supported frequencies cover the L1/E1 band, the L2 band and the L5/E5a band. All signals are jointly processed on a LEON-FT micro-processor embedded in the AGGA-4 chip. In total, dual-frequency tracking of up to 18 GPS or Galileo satellites is supported, including $\mathrm{P}(\mathrm{Y})$ tracking for up to 8 GPS satellites.

An overview of supported signals and tracking modes is provided in Table 1 along with the corresponding designations of the receiver independent exchange format (RINEX; Romero 2020) for GNSS measurements. In case of the modernized signals, PODRIX observations are exclusively based on tracking of the pilot component, while the navigation data bits are obtained with a slaved correlator for the data component. For the Galileo E1 open service signal, only the $\mathrm{BOC}(1,1)$ binary offset carrier is considered in the code replica generation, while the $\operatorname{BOC}(6,1)$ contribution is 
ignored (Roselló et al. 2012). This results in a small loss of carrier-to-noise density ratio, $C / N_{0}$, compared to a tracking of the actual composite BOC (CBOC) modulation, which amounts to roughly $1 \mathrm{~dB}$ and can well be tolerated in the present application.

In view of various resource limitations, the set of jointly tracked GPS signals in the PODRIX receiver is kept to the necessary minimum for dual-frequency navigation, using a configurable choice of signals for the individual blocks of GPS satellites. In the default scheme adopted for the nominal receiver during the commissioning phase, C/A and L1/L2 $\mathrm{P}(\mathrm{Y})$-code tracking is used for Block IIR satellites, while the C/A and L2C signals are tracked for IIR-M, IIF, and GPS III satellites. No use is made of L5 observations despite their superior characteristics, since these are only available for half of the current GPS constellation and would have resulted in different frequency pairs (L1/L5 vs L1/L2) across the set of tracked satellites. For Galileo, the pilot signals on E1 and E5a are tracked for all healthy satellites in nominal orbit, but tracking of space vehicle numbers SVN E201 and E202 in eccentric orbits is currently not supported due the lack of Galileo almanac data for visibility prediction. On average, GPS P(Y), GPS C/A+L2C, and Galileo E1+E5a observations were available for approximately 1.6, 6.2, and 6.3 satellites in early 2021 at a $10^{\circ}$ elevation mask.

The use of different observation types within the GPS constellation poses specific challenges for a proper POD processing and requires careful consideration of inter-signal biases. While biases related to the GPS satellites themselves can be considered based on corresponding products made available through the International GNSS Service (IGS; Johnston et al. 2017), user-related equipment biases need to be corrected based on dedicated calibrations or treated as unknown solve-for parameters. Code biases in the PODRIX receivers and antenna were found to cause a bias of roughly $4 \mathrm{~m}$ in the ionosphere-free (IF) combination of $\mathrm{C} / \mathrm{A}+\mathrm{L} 2 \mathrm{C}$ observations as compared to $\mathrm{L} 1 / \mathrm{L} 2$ $\mathrm{P}(\mathrm{Y})$ on Sentinel-6A, which reflects itself in a corresponding inconsistency of the estimated receiver clock offsets or pseudorange residuals. Likewise, these biases affect the Hatch-Melbourne-Wuebbena combination (HMW; Hatch 1983; Melbourne 1985; Wübbena 1985) and therefore impact the fixing of integer ambiguities in the processing of GPS carrier phase measurements.

Calibrations of temperature-dependent code biases for all signal types have been performed by the manufacturer for the individual flight units of the PODRIX receivers and are made available as part of the instrument calibration database (ICDB). Along with measurements of the front-end temperature provided in the receiver telemetry, these data were used by the authors to correct the code measurements prior to the generation of RINEX observation files for the POD processing from the raw GNSS receiver data. Frontend temperatures
Table 2 Signal-specific group delay corrections of raw pseudoranges (in [m]) for the nominal PODRIX receiver of Sentinel-6A as a function of the frontend temperature

\begin{tabular}{llrrrr}
\hline Signals & & \multicolumn{2}{c}{ F/E temperature $\left[{ }^{\circ} \mathrm{C}\right]$} & \multicolumn{1}{c}{$\begin{array}{l}\text { Emp. } \\
\text { corr. }\end{array}$} \\
\cline { 3 - 5 } & & 8.8 & 18.9 & 28.5 & \\
\hline GPS & 1C & 0.000 & 0.000 & 0.000 & \\
& 1W & 0.559 & 0.945 & 1.228 & 0.22 \\
& 2W & 0.395 & 1.036 & 1.371 & -0.12 \\
& 2L & 1.363 & 2.159 & 2.763 & \\
& 5Q & -0.520 & 0.167 & 0.483 & \\
Galileo & 1C & 0.000 & 0.000 & 0.000 & \\
& 5Q & -0.799 & -0.589 & -0.609 & \\
& & & & &
\end{tabular}

Intermediate values are obtained by linear interpolation. In addition, empirical offsets for aligning observations of civil and encrypted GPS signals are given based on the analysis of flight data

in the nominal receiver were found to vary between daily means of about $15-20^{\circ}$ that partly relate to the Sun's elevation angle above the orbital plane ( $\beta$-angle) but also show stepwise variations attributed to operational changes of onboard equipment. In addition to the long-term changes, superimposed orbital variations of about $\pm 1^{\circ}$ may be observed in response to the varying Sun illumination. An overview of ICDB corrections for the temperature range of interest is provided in Table 2. Application of the ICDB corrections greatly reduces the inconsistencies in the IF and HMW combinations for the two signal groups, but does not provide a full correction. Based on an analysis of flight data from the first month, additional empirical corrections of $0.22 \mathrm{~m}$ and $-0.12 \mathrm{~m}$ are therefore applied to the $\mathrm{L} 1$ and $\mathrm{L} 2 \mathrm{P}(\mathrm{Y})$ code observations, respectively. It is presently unclear whether these additional biases relate to imperfections of the pre-flight receiver calibration, incompatible temperature data, or biases outside the actual receiver, i.e., cables, low noise amplifier, and antenna. Further monitoring and adjustment of the empirical biases throughout the mission is therefore suggested to facilitate Sentinel-6A POD based on PODRIX GNSS measurements.

Concerning carrier phase observations, L1 phase measurements in the PODRIX receiver are consistently obtained from C/A-code tracking in the AGGA-4 correlator. For L2, in contrast, phase measurements are based on either L2C tracking in the AGGA-4 or semi-codeless $\mathrm{P}(\mathrm{Y})$ tracking in the AGGA2 chip. Both signals are transmitted in phase-quadrature by the modernized GPS satellites and a $-1 / 4$ cy adjustment of the $\mathrm{L} 2 \mathrm{C}$ phase is required for alignment to the $\mathrm{P}(\mathrm{Y})$ phase reference in RINEX observations files (Romero 2020). However, additional fractional phase biases may be observed in the PODRIX L2 phase measurements which can most likely be attributed to different delays in the internal clock signals of the two chipsets. While such biases do not affect a POD processing based on float-ambiguity estimation, they inhibit a seamless use of mixed signal types in carrier phase 
Table 3 Phase center offsets from antenna reference point of Sentinel$6 \mathrm{~A}$ antenna including manufacturer calibration and adjusted reference values for precise orbit determination

\begin{tabular}{llll}
\hline Type & $x[\mathrm{~mm}]$ & $y[\mathrm{~mm}]$ & $z[\mathrm{~mm}]$ \\
\hline RUAG (ref) & +0.0 & +0.0 & +97.0 \\
RUAG mean L1/E1 & -0.7 & -0.7 & +93.6 \\
RUAG mean L2 & -0.6 & -0.8 & +89.2 \\
RUAG mean L5/E5a & -0.8 & -0.9 & +75.3 \\
RUAG mean IF(L1,L2) & -0.9 & -0.5 & +100.4 \\
RUAG mean IF(E1,E5a) & -0.6 & -0.4 & +116.7 \\
POD IF(L1,L2) & 0 & 0 & +75 \\
POD IF(E1,E5a) & 0 & 0 & +93 \\
\hline
\end{tabular}

All values apply for the antenna of the nominal receiver and refer to the antenna reference frame, where $+z$ describes the nominal boresight direction and $+x$ is aligned with the $+x$-axis of the spacecraft frame

ambiguity resolution and need to be corrected as part of the measurement generation process.

Since L2 $\mathrm{P}(\mathrm{Y})$ and $\mathrm{L} 2 \mathrm{C}$ carrier phase observation are never obtained concurrently for a given GPS satellite, we adjusted the bias of the $\mathrm{L} 2 \mathrm{C}$ phase measurements relative to those of $\mathrm{P}(\mathrm{Y})$ tracking in a short baseline test with the redundant receiver of Sentinel-6A while that receiver was temporarily operated in a $\mathrm{P}(\mathrm{Y})$-only configuration on November 28 , 2020. After accounting for the known antenna baseline and line-of-sight directions, double-difference observations for pairs of satellites with mixed carrier phase types can directly be used to estimate the $\mathrm{L} 2 \mathrm{C}-\mathrm{P}(\mathrm{Y})$ phase bias of the nominal receiver. While potentially affected by antenna phase pattern differences and local multipath, the resulting estimate of $0.075 \mathrm{cy}$ was found to agree well with that of a laboratory test $(0.073 \mathrm{cy})$ of a PODRIX engineering model and a geodetic reference receiver. Compared to an expected quarter cycle offset, the actual L2C phase bias amounts to roughly $1 / 3 \mathrm{cy}$ and clearly needs to be taken into account for all forms of carrier phase-based positioning with ambiguity resolution.

\section{Antenna calibration}

The PODRIX receiver is operated with a triple-band (L1/E1, L2, L5/E5a) patch excited cup antenna (PEC; Öhgren et al. 2011), with choke ring for improved multipath suppression (Fig. 2).

Nominal values of the phase center relative to the mechanical antenna reference point (ARP) can be obtained from pre-flight anechoic chamber calibrations of the phase pattern for individual frequencies relative to a reference phase center offset (PCO) and are provided by the manufacturer as part of the ICDB. Based on these data, mean PCOs have been adjusted that minimize the deviations from a spherical wave- front over the $+z$ hemisphere. The resulting PCOs exhibit horizontal offsets of less than $1 \mathrm{~mm}$ from the geometric center, but notable differences may be observed in boresight direction for the individual frequencies (Table 3). For the ionosphere-free combination of L1/L2 and E1/E5a carrier phases, the effective PCOs differ by $16 \mathrm{~mm}$ in $z$-direction. Phase variations (PVs) with respect to the mean PCOs as measured in the pre-flight calibration exhibit a high level of rotational symmetry around the boresight axis and are confined to less than $\pm 2 \mathrm{~mm}$ for elevations above $10^{\circ}$ (Fig. 3).

Similar to many other LEO missions with dual-frequency GNSS receivers, pronounced phase pattern distortions may, however, be observed for the GNSS antennas after integration into the spacecraft (Montenbruck et al. 2008, 2009) as a result of local multipath and other near-field effects. In case of Sentinel-6A, these may exhibit up to 10-times higher amplitudes than phase variations of the standalone antenna and necessitate a complementary in-flight calibration for precise modeling of the carrier phase observation in the POD process. Following Jäggi et al. (2009), we made use of a residual stacking approach to obtain distinct L1/L2 and E1/E5a antenna phase patterns from GPS and Galileo observations collected over a 35 days period from day of year (DOY) 2020/354 to 2021/022. The resulting patterns are shown in Fig. 3 and reveal a patchy structure with RMS amplitudes of about $4 \mathrm{~mm}$ and peaks up to 5 times this value over the $80^{\circ}$ boresight angle cone considered for tracking by the receiver.

The patterns refer to conventional values of the PCOs listed in Table 3, which were initially adjusted to remove the mean vertical offset between kinematic and reduceddynamic orbit determination solutions within a $1 \mathrm{~mm}$ tolerance. We note that the resulting calibration is implicitly tied to the employed force models and slightly different results may, for example, be obtained when using different macromodels for solar and Earth radiation pressure. While the $18 \mathrm{~mm}$ difference of the adjusted L1/L2 and E1/E5a $z$-PCOs is close to that of the pre-flight chamber calibration results, the adjusted $z$-PCOs themselves are roughly $25 \mathrm{~mm}$ smaller than suggested by the factory calibration.

Considering possible sources of this discrepancy, it may be noted that the radial component of the position vector in a dynamic or reduced-dynamic orbit determination is essentially determined by the employed force models. For near-circular LEO satellite orbits, a change of $\Delta a_{\mathrm{R}}$ in the modeled radial acceleration within the POD process causes a difference of

$\Delta r_{\mathrm{R}}=-\frac{1}{3}\left(\frac{T}{2 \pi}\right)^{2} \Delta a_{\mathrm{R}}$

in the estimated orbital radius, where $T$ denotes the period of period of revolution (Montenbruck et al. 2018). For the specific case of Sentinel-6A, a $10 \mathrm{~nm} / \mathrm{s}^{2}$ increase in the radial 
Fig. 3 Zero-mean antenna patterns of the nominal Sentinal-6A GNSS antenna for ionosphere-free L1/L2 (top) and E1/E5a (bottom) carrier phase observations as obtained from the pre-flight chamber calibration (left) and from carrier phase residuals in the POD process (right)



acceleration lowers the resulting orbit by about $3.8 \mathrm{~mm}$. The kinematic position, in contrast, is determined by the orbital radius of the GNSS satellites, the assumed transmit antenna phase center offset and pattern, and, finally, the assumed offset of the receiver ARP from the LEO center of mass (CoM).

Models and standards used in the present analysis are summarized in Table 4. Even though the modeling of nongravitational forces introduces some uncertainties in the dynamical leveling of the CoM trajectory, experience from past LEO missions and cross comparison with other POD centers shows that associated uncertainties in mean height are typically less than $5 \mathrm{~mm}$ (Kobel et al. 2019; Fernández et al. 2019). As such, force model deficiencies are unlikely to explain the observed $\approx 25 \mathrm{~mm}$ PCO difference between manufacturer and in-flight calibration. GPS satellite orbits and transmit antenna data for the period of interest are aligned to the IGS 14b reference frame and provide consistent kinematic positions of the receiver antenna phase center in this frame. In case of Galileo, possible frame height inconsistencies of 6-7 $\mathrm{mm}$ may arise from the use of manufacturer-calibrated transmit antenna patterns in the generation of Galileo orbit and clock products (Villiger et al. 2020; Huang et al. 2021) but would only explain potential discrepancies in the relative location of the GPS L1/L2 and Galileo E1/E5a PCOs. However, these amount to $16 \mathrm{~mm}$ and $18 \mathrm{~mm}$ for chamber and inflight calibrations (Table 3), respectively, and are thus consistent within $2 \mathrm{~mm}$.

\section{POD concepts and models}

For Sentinel-6A orbit determination, we make use of a reduced-dynamic approach (Wu et al. 1991), which combines a state-of-the-art a priori model of the spacecraft dynamics with the estimation of empirical accelerations to overcome prevailing uncertainties in the modeling of nongravitational forces. The processing with DLR's GNSS High precision Orbit determination Software Tools (GHOST) builds on basic concepts and algorithms for reduced-dynamic orbit determination and single-receiver ambiguity fixing as described in Montenbruck et al. (2005) and Montenbruck et al. (2018). In preparation for Sentinel-6 and other upcoming space missions with advanced GNSS receivers, the software has been extended to support multiple constellations as well as mixed signal types within an individual constellation. Among others, this includes support for signal-specific code biases as well as the capability to adjust epoch-wise inter-system biases (ISBs).

An overview of the employed models and data is given in Table 4. Satellite-specific data including GNSS antenna and LRA positions and orientations, body dimensions and sur- 
Table 4 Models and data sets for Sentinel-6A precise orbit determination and SLR validation

GNSS Measurements

GNSS observations

GNSS orbit and clocks

GNSS satellite biases

GNSS satellite antennas

GNSS satellite attitude

GNSS block types

S6A GNSS antenna

S6A attitude

Reference frame

Phase wind-up
Undifferenced GPS L1/L2 and Galileo E1/E5a pseudorange and carrier phase range (Table 1);

30 s sampling, daily arcs

CNES-CLS “GRGOMGXFIN” GPS/Galileo product (Loyer et al. 2012; Katsigianni et al. 2019),

CODE “_M” rapid GPS/GLONASS/Galileo product from middle day of 3 day solutions (Dach et al. 2020;

Prange et al. 2020);

30 s sampling

CNES-CLS wide-lane satellite biases (Loyer et al. 2012; Katsigianni et al. 2019) and CAS DCBs Wang et al. (2016);

CODE “_M” rapid observable-specific biases for GPS, GLONASS, Galileo (Schaer et al. 2021; Dach et al. 2020)

IGS igs14.atx antenna model (week 2136 Rebischung and Schmid 2016) with estimated L1/L2 PCOs and patterns for GPS Block IIR, IIR-M, and IIF satellites, manufacturer PCOs for GPS III (L1, L2) satellites, and manufacturer PCOs/PVs for Galileo satellites

Nominal yaw-steering

IGS satellite metadata file (week 2144)

antenna reference point $(2.475 \mathrm{~m}, 0.000 \mathrm{~m},-1.080 \mathrm{~m})$ (Cullen 2021); PCO+PV from inflight calibration

Quaternions (measured)

IGb14 (Rebischung 2020)

Modeled (Wu et al. 1993)

Orbit Model

Earth gravity field

Luni-solar gravity

Solid Earth and pole tides

Ocean tides

Relativity

Spacecraft parameters

Solar radiation pressure

Earth radiation pressure

Atmospheric forces

Maneuvers

Empirical acceleration

Reference frame

Earth orientation

Numerical integration
GOCO03S (Mayer-Gürr et al. 2012) up to order and degree 70; rate terms $\dot{C}_{20}, \dot{C}_{21}, \dot{S}_{21}$,

point-mass model; analytical series of luni-solar coordinates

IERS2003

FES2004-TEG4 (Lyard et al. 2006)

Post-Newtonian correction

Mass $(1180.6 \mathrm{~kg})$ and CoM position $(1.533 \mathrm{~m},-0.007 \mathrm{~m}, 0.037 \mathrm{~m})$ after December 16,2020$)$ from spacecraft operator database; 6 panel macro-model

Spacecraft macro-model; conical Earth shadow model

Spacecraft macro-model; polynomial/harmonic approximation (Knocke et al. 1988) of CERES Earth radiation data (Priestley et al. 2011), 15 rings/135 segments approximation of sub-satellite region

Macro-model with Sentman's formulation of drag and lift (Sutton 2009; Doornbos 2012);

NRLMSISE-00 density model (Picone et al. 2002), NOAA/SWPC solar flux and geomagnetic activity data (ftp:// ftp.swpc.noaa.gov/pub/indices/)

Constant thrust in RTN direction; 5 maneuvers from November 27 to December 18, 2020; burn time and approximate $\Delta v$ from spacecraft operator

Piecewise constant accelerations in RTN direction; 10 min intervals

ICRF

IERS1996; IGS final EOPs; center-of-mass/center-of-figure offset

Self-starting variable-order variable stepsize multistep method (Shampine and Gordon 1975)

Estimation

Filter

Estimation parameters

Stochastic models
Batch least-squares estimation

Epoch state vector, empirical accelerations and maneuvers, epoch-wise clock offsets and inter-system biases, phase ambiguities

White observation noise, elevation-independent weighting; zero a priori values and configurable standard deviation of empirical accelerations

SLR

Station coordinates

SLRF2014 (https://cddis.nasa.gov/archive/slr/products/resource/ ${ }^{\dagger}$ )

Solid Earth and pole tides 
Table 4 continued

SLR

Ocean tide loading

Atmospheric pressure loading

Tropospheric refraction

Relativity

LRR phase correction
GOT00.2 (Ray 1999)

TU Vienna model (Wijaya et al. 2013; VMF 2021)

IERS2010 (Mendes and Pavlis 2004)

Space-time curvature correction

Optical center at $(1.6248 \mathrm{~m},-0.4006 \mathrm{~m}, 0.6648 \mathrm{~m})$; azimuth- and nadir-angle-dependent range correction

${ }^{\dagger}$ Access to CDDIS repository requires prior registration and NASA EOSDIS login

face properties, time-dependent mass and CoM locations, as well as the spacecraft attitude were provided by the manufacturer and the Sentinel-6A operations team. Auxiliary GNSS data including precise orbits and clocks, as well as code and phase biases were obtained from the IGS or its individual analysis centers. For comparison of multi-constellation processing and ambiguity resolution performance, independent POD solutions were generated using products from the Centre National d'Études Spatiales/Collecte Localisation Satellites (CNES/CLS) and the Center for Orbit Determination in Europe (CODE).

The observation model takes into account conventional GNSS transmit antenna offsets and patterns consistent with the employed orbit and clock products. In accord with established IGS standards, both CNES/CLS and CODE clock products refer to a ionosphere-free combination of L1/L2 $\mathrm{P}(\mathrm{Y})$-code measurements for GPS and E1/E5a measurements for Galileo. While this enables direct processing of the respective PODRIX measurements, satellite-specific code biases need to be applied in the modeling of GPS L1 C/A and L2C observations. When working with CODE orbit and clock products, we made use of observable-specific biases from the associated CODE bias product, while differential code biases (DCBs) provided by the Chinese Academy of Science were used with the CNES/CLS clock products.

Single-receiver carrier phase ambiguity resolution is performed using a wide-lane/narrow-lane fixing strategy as described in Laurichesse et al. (2009) and Montenbruck et al. (2018). The processing is based on individual passes, i.e., arcs of continuous and cycle-slip-free carrier phase tracking. In a first step, an average value of the HMW combination is determined for each pass from the dual-frequency pseudorange and phase observations. Aside from measurement noise, it represents the sum of the pass-specific wide-lane ambiguity as well as the fractional receiver and satellite wide-lane biases. Taking into account the known satellite biases, integer rounding is used to separate the fractional bias, which is common to all passes, and to determine the integer-valued wide-lane ambiguities for all passes.

As part of the orbit determination a float-valued estimate $b_{i}$ of the ambiguity of the ionosphere-free, dual-frequency combination is obtained for each pass $i$. Making use of the fixed wide-lane ambiguities, $b_{i}$ can be expressed as the sum of a receiver-specific fractional phase bias, which is common to all passes, a satellite-specific fractional phase bias, and a pass-specific, integer-valued $N_{1, i}$ ambiguity on the L1 (or E1) frequency. The integer ambiguities of all passes are then obtained through rounding of between-pass differences of the ambiguities $b_{i}$ after accounting for the known satellite biases. Subsequently, the fixed wide- and narrow-lane ambiguities are introduced as soft constraints for the estimation of $b_{i}$ in subsequent iterations of the orbit determination process. This is accomplished by adding the between-passes differences of the ionosphere-free carrier phase ambiguities $b_{i}-b_{j}$ computed from the integer-valued wide- and narrowlane ambiguities as pseudo-observations with small (0.1 mm) a priori standard deviation to the overall normal equations.

Depending on the orbit/clock product supplier, wide-lane biases for the GNSS satellites are either provided directly as an independent product (CNES/CLS) or can be computed from the observable specific code and phase biases in the generic bias product (CODE). Fractional phase biases of the GNSS satellites may be lumped into the clock estimates (CNES/CLS) or provided as part of the observable specific bias product. As discussed in Banville et al. (2020), both representations are fully equivalent and can be used interchangeably provided that a consistent set of clock and bias products is used. In case of GPS, receiver-specific biases between L1 C/A and L1 P(Y) observations as well as L2C and L2 $\mathrm{P}(\mathrm{Y})$ observations have been removed as part of the PODRIX measurement generation to the extent possible. As such, ambiguity resolution can be performed across all satellites of the GPS constellation irrespective of the particular signals tracked.

The trajectory model for Sentinel-6A orbit determination makes use of established models for Earth gravity including tidal contributions, luni-solar gravitation and relativistic corrections in a post-Newtonian approximation. Surface forces due to solar and Earth radiation pressure (SRP, ERP) as well as drag are described through a macro-model taking into account the principal body shape and dimensions (Table 5). Optical surface properties comprise the fractions of absorbed $(\alpha)$ as well as diffusely $(\delta)$ and specularly $(\rho)$ reflected photons in the visible and infrared region, which are used to 
Table 5 Sentinel-6A macro-model. Adapted from Cullen (2021)

\begin{tabular}{|c|c|c|c|c|c|c|c|c|}
\hline Element & Surface normal & Area $\left[\mathrm{m}^{2}\right]$ & $\alpha_{\mathrm{VIS}}$ & $\delta_{\mathrm{VIS}}$ & $\rho_{\mathrm{VIS}}$ & $\alpha_{\mathrm{IR}}$ & $\delta_{\mathrm{IR}}$ & $\rho_{\mathrm{IR}}$ \\
\hline Body $+X$ (front) & $(+1.000,+0.000,+0.000)$ & 3.5 & 0.47 & 0.04 & 0.49 & 0.76 & 0.04 & 0.20 \\
\hline Body $-X$ (back) & $(-1.000,+0.000,+0.000)$ & 3.5 & 0.43 & 0.12 & 0.45 & 0.78 & 0.04 & 0.18 \\
\hline Body $+Z$ (bottom) & $(+0.000,+0.000,+1.000)$ & 15.5 & 0.57 & 0.08 & 0.35 & 0.77 & 0.12 & 0.11 \\
\hline Body $-Z$ (top) & $(+0.000,+0.000,-1.000)$ & 2.0 & 0.35 & 0.03 & 0.62 & 0.77 & 0.01 & 0.22 \\
\hline Solar panel (starboard) & $(+0.000,+0.616,-0.788)$ & 8.9 & 0.86 & 0.14 & 0.00 & 0.80 & 0.20 & 0.00 \\
\hline Solar panel (portside) & $(+0.000,-0.616,-0.788)$ & 8.9 & 0.86 & 0.14 & 0.00 & 0.80 & 0.20 & 0.00 \\
\hline Body $+Y$ (bottom structures) & $(+0.000,+1.000,+0.000)$ & 1.0 & 0.43 & 0.14 & 0.43 & 0.75 & 0.12 & 0.13 \\
\hline Body $-Y$ (bottom structures) & $(+0.000,-1.000,+0.000)$ & 1.0 & 0.43 & 0.14 & 0.43 & 0.75 & 0.12 & 0.13 \\
\hline
\end{tabular}

See Fig. 1 for the orientation of the spacecraft body system

compute the net radiation pressure force for each surface element as a function of the incident radiation (Milani et al. 1987). The respective values are based on manufacturer data for mylar insulation, optical surface reflectors and solar cells and take into account the share of individual materials for each of the panel (Cullen 2021). In our macro-model, diffuse re-emission of absorbed photons in both the visible and infrared region is assumed for all surfaces including the solar panels. This is different from common practice (Cerri et al. 2010), where diffuse re-emission is only considered for surfaces covered by thermal insulation layers but not assumed for solar panels. However, the SRP contribution of the large solar panels in the a priori macro-model without considering such re-emission is found to be roughly one-third too small compared to the observed accelerations. This empirical evidence suggests that the standard model is mainly applicable for solar panel "wings" where thermal re-emission from the front and backside is largely balanced. Sentinel-6, in contrast, makes use of surface mounted panels where additional thermal insulation is likely to create an asymmetric re-radiation toward the sun-lit hemisphere.

The employed macro-model represents an obvious simplification of the actual spacecraft geometry, but, nevertheless, captures the dominating variation of radiation pressure forces under the varying Sun-incidence angles. Among others, contributions of the AMR-C dish antenna with a cross section of about $1 \mathrm{~m}^{2}$ as well as the concave regions between the satellite body and the back-side of the outer solar panels are not considered. A coarse approximation of radiation pressure due to structures on the nadir-looking side (including the conic cover of the altimeter antenna, the DORIS antenna, and other elements) is obtained by considering two "panels" with $\pm y$ surface normals and a $1 \mathrm{~m}^{2}$ cross section in the macromodel. The model simplifications are largely motivated by the computational complexity of a full-featured ray-tracing model that would be needed to account for self-shadowing or multiple reflections, but is also justified by the use of approximate optical coefficients with a presumed uncertainty of 0.1 . Within the POD, these modeling deficiencies are compen- sated by the adjustment of piece-wise constant empirical accelerations in radial (R), along-track (T) and cross-track (N) direction over intervals of ten minutes. For Sentinel-6A, a priori values of zero and standard deviations of $4 \mathrm{~nm} / \mathrm{s}^{2}$, $8 \mathrm{~nm} / \mathrm{s}^{2}$, and $15 \mathrm{~nm} / \mathrm{s}^{2}$ are adopted for the three axes. These constraints are chosen in accord with the observed amplitude of estimated accelerations, except for the radial component, which shows a notable coupling with the along-track accelerations. At the given magnitude of the a priori constraints, the estimated empirical accelerations may absorb systematic errors in the assumed antenna offsets from the CoM at a level of about $1.5 \mathrm{~mm}$ in radial direction (Eqn. (1)) and $15 \mathrm{~mm}$ in cross-track direction (Sect. 5.4).

A batch least-squares estimator is used for the Sentinel$6 \mathrm{~A}$ orbit determination. Next to the receiver clock offsets and Galileo-GPS inter-system biases, the adjusted parameters comprise the initial state vector, force model scale factors, and empirical accelerations, as well as carrier phase ambiguities. Similar to the receiver clock offsets, ISBs are also estimated as epoch-wise parameters without a priori constraints to account for temperature related, orbital variations as well as irregular phase slips in the L1/E1, L2, and L5/E5a frontends of the PODRIX receiver. At a $30 \mathrm{~s}$ sampling roughly 5800 clock and ISB parameters need to be adjusted for a $24 \mathrm{~h}$ data arc, which drives the overall dimension of the least squares system. Using pre-elimination, the resulting normal equations can, however, be reduced to the dimension of orbit and ambiguity parameters. Considering 10 min intervals for the empirical accelerations and about 550 passes for roughly 50 GPS and Galileo satellites observed in each revolution, a total of 1000 parameters are determined in the reduced normal equations.

\section{Results and validation}

Making use of the methodology described above, precise orbits for the Sentinel-6A satellite were computed over a 6-month time span starting with the first transmission of 
science telemetry on November 27, 2020. Throughout this period, GNSS data were collected with the "nominal" (primary) PODRIX receiver, which was connected to the DORIS ultrastable oscillator (USO) and configured for "mixed," block-specific GPS signal types.

Observation data in RINEX format were generated from the raw receiver data using code and phase bias corrections as described in Sect. 2. The receiver time scale used in the generation of the pseudorange and phase measurements was related to the instrument measurement time (IMT), which measures time based on the nominal oscillator frequency, by a third-order polynomial in IMT that minimizes the difference of receiver time and GPS time over the daily data arc. In this way, clock offsets are minimized while retaining a traceable link to the DORIS USO frequency.

As part of the measurement generation, corrections for occasional half-cycle phase slips occurring concurrently for all observations in a specific frequency band were corrected manually after initial inspection and analysis. In total, 30 such events were encountered in the 6-month data arc with an increased number of events found between end of December 2020 and mid-January 2021 as well as the second half of March 2021. All such phase slips occurred in the vicinity of the South Atlantic Anomaly (SAA), where the inner Van Allen radiation belt comes close to the surface of the Earth (Hess 1968). They may thus be interpreted as radiationinduced single-event effects (SEEs) in the receiver frontend, even though the detailed mechanism of their occurrence still needs further investigation. While uncritical for floatambiguity POD solutions, the phase slips impact the wideand narrow-lane ambiguity and need to be repaired for proper ambiguity resolution on the affected days.

\subsection{Measurement-related quality indicators}

Independent POD solutions were computed using combined GPS/Galileo orbit and clock products from the CODE and CNES/CLS analysis centers of the IGS. Internal quality checks of these solutions are provided by code and carrier phase residuals, as well as the success rate of the ambiguity solution, which provide a measure of the data quality and the overall goodness of fit. Results for the combined GPS/Galileo solutions as obtained with the two sets of GNSS products are summarized in Tables 6 and 7. The Galileo measurements clearly outperform those of GPS with RMS residuals that are roughly $30-50 \%$ smaller. Observations based on the civil C/A and L2C signals of GPS show slightly higher residuals than those of semi-codeless $\mathrm{P}(\mathrm{Y})$-code tracking for the tracking loop bandwidth adopted in the PODRIX receiver, but can be expected to offer increased robustness and reduced sensitivity to ionospheric scintillation (van den IJssel et al. 2016).

In comparing PODs based on CODE products with those based on CNES/CLS products, an obvious increase of the
$\mathrm{C} 1 \mathrm{C} / \mathrm{C} 2 \mathrm{~L}$ code residuals level may be observed. This degradation is not related to the measurements themselves, but can be attributed to an inferior quality of CODE pseudorange biases for the L2C signal. Other than the CAS DCBs, which originate from a large $\mathrm{L} 2 \mathrm{C}$-capable receiver network, CODE's observable specific biases (Villiger et al. 2019) for the $\mathrm{L} 2 \mathrm{C}$ signals are presently derived from only a small subset of IGS stations still delivering their observations in the old RINEX v2 format. However, the reduced quality of the CODE OSBs has only a negligible effect on the wide-lane ambiguity determination from the HMW combination and does not impact the overall success rate of wide-lane and $N_{1}$-ambiguity resolution. Overall, a better than $92 \%$ fixing rate is obtained in $95 \%$ of all days over the data analysis period (Table 7).

\subsection{Clock performance}

The DORIS USO is a highly accurate and stable ovencontrolled quartz oscillator (Debaisieux et al. 1985; Auriol and Tourain 2010; Jayles et al. 2016), which serves as the common frequency reference for the DORIS instrument as well as the main PODRIX receiver. Starting from an initial value of about $-3.2 \mathrm{~ns} / \mathrm{s}$, the USO drift relative to GPS time decreased to roughly $-0.1 \mathrm{~ns}$ over the first 6 months of operation.

Based on a "one-way carrier phase" (OWCP) analysis (Gonzalez and Waller 2007) of detrended $1 \mathrm{~Hz}$ PODRIX carrier phase observations an upper limit of the USO's Allan deviation ranging from $5.3 \times 10^{-12} \mathrm{~s} / \mathrm{s}$ at time intervals of $1 \mathrm{~s}$ down to $1.2 \times 10^{-13} \mathrm{~s} / \mathrm{s}$ at $100 \mathrm{~s}$ can be derived. These values are governed by thermal noise of the GNSS measurements and possibly short-term clock stability of the tracked GNSS satellites. As such, they can only provide an upper limit on the actual USO performance, which is considered better by up to one order of magnitude. Nevertheless, the OWCP analysis shows that no cross talk between the internal PODRIX oscillator and the external DORIS USO can be recognized, which was previously found to cause a few-cm oscillation of the clock signal with a $1.5-3 \mathrm{~Hz}$ beat frequency in the GPS receiver of the Sentinel-3A mission (Jalabert and Mercier 2018).

Clock variations over time scales of up to one day are illustrated Fig. 4, which shows the offset of the detrended USO time with respect to GPS time as obtained in the POD for a selected day in January 2021. Here, periodic relativistic variations (Kouba 2019) related to the eccentricity and Earth oblateness have been removed from the apparent clock. These vary with one and two cycles per revolution, respectively, and exhibit amplitudes at the level of one decimeter for the Sentinel-6A orbit. Local peaks in the clock offset relate to SAA passages where the oscillator experiences small frequency jumps in response to ionizing radiation. In between 
Table 6 Residuals statistics

\begin{tabular}{lllllll}
\hline GNSS product & Pseudorange & & \multicolumn{2}{l}{ Carrier phase } \\
\cline { 2 - 6 } & $\begin{array}{l}1 \mathrm{~W} / 2 \mathrm{~W} \\
{[\mathrm{~m}]}\end{array}$ & $\begin{array}{l}1 \mathrm{C} / 2 \mathrm{~L} \\
{[\mathrm{~m}]}\end{array}$ & $\begin{array}{l}1 \mathrm{C} / 5 \mathrm{Q} \\
{[\mathrm{m}]}\end{array}$ & $\begin{array}{l}1 \mathrm{~W} / 2 \mathrm{~W} \\
{[\mathrm{~mm}]}\end{array}$ & $\begin{array}{l}1 \mathrm{C} / 2 \mathrm{~L} / 5 \mathrm{Q} \\
{[\mathrm{mm}]}\end{array}$ \\
\hline CNES/CLS & 0.57 & 0.64 & 0.37 & 5.2 & 6.1 & 4.3 \\
CODE & 0.54 & 0.76 & 0.36 & 5.2 & 6.1 & 4.3 \\
\hline
\end{tabular}

The median value of daily RMS residuals for the ionosphere-free combination of pseudorange and carrier phase observations in ambiguity fixed reduced-dynamic POD is provided, where individual observations are identified by their RINEX designations

Table 7 Ambiguity resolution statistics for wide-lane $\left(N_{\mathrm{WL}}\right)$ and $N_{1}$ ambiguities

\begin{tabular}{lllll}
\hline GNSS product & $N_{\mathrm{WL}, \mathrm{GPS}}$ & $N_{\mathrm{WL}, \mathrm{Gal}}$ & $N_{1, \mathrm{GPS}}$ & $N_{1, \mathrm{Gal}}$ \\
\hline CNES/CLS & 96.0 & 99.6 & 92.6 & 95.7 \\
CODE & 96.3 & 99.6 & 92.7 & 95.6 \\
\hline
\end{tabular}

All values are given in $\%$ and denote the 5 th percentile value, implying that $95 \%$ of all solutions have an ambiguity success rate higher than the given value
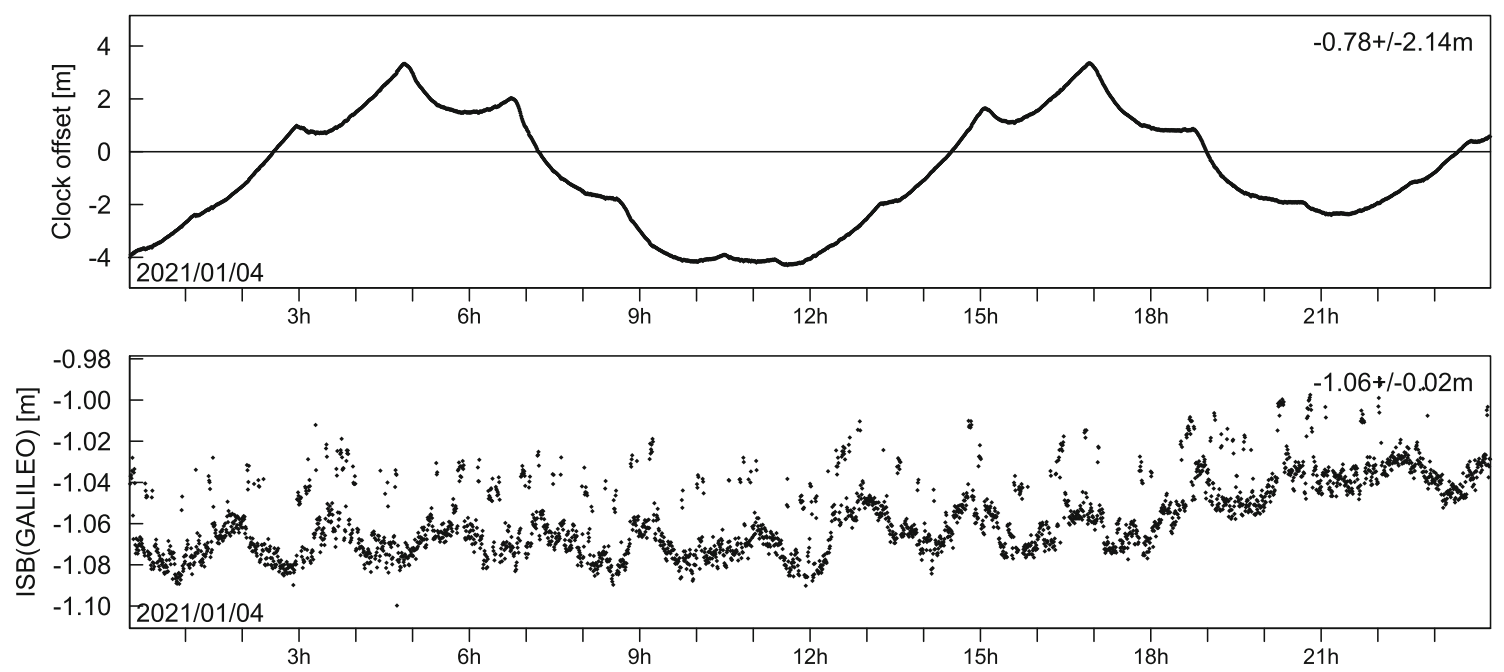

Fig. 4 Estimated Sentinel-6A clock offsets (top) and Galileo-GPS inter-system biases (bottom) for January 42021 based on CNES/CLS clock products

those peaks, a largely parabolic variation of the clock offset may be noticed. As discussed in Štěpánek et al. (2020), GNSS-based observations of the radiation-induced USO frequency variation will allow to correct observations of the DORIS instrument on Sentinel-6A and obtain improved position estimates of DORIS beacons.

Concerning absolute onboard time synchronization, it may be noted that the estimated clock offsets depend on the GPS time realization in the respective GNSS clock product. Here, differences at the $0.3 \mathrm{~m}$ (1 ns) level may typically be observed between solutions obtained with either CNES/CLS or CODE products.

Figure 4 also illustrates the temporal variation of the Galileo-GPS inter-system biases as estimated in the POD process. While the absolute value and the overall drift of the estimated ISB depends again on the employed GNSS clock product, short-term variations are consistently reflected in all POD solutions. Aside from orbit-periodic variations attributed to temperature-related bias changes, irregular ISB jumps at the level of about $3 \mathrm{~cm}$ may be noticed. These have previously been identified in PODRIX ground tests and are related to phase jumps in the local oscillators for signal downconversion in the three frontends of the receiver. Depending on the specific frequency band (L1/E1, L2, L5/E5A) affected by a jump, discontinuities will arise in the estimated receiver clock offset and/or the ISB. While these glitches put a natural limit to the observability of the DORIS frequency stability, they do not affect the DORIS operation itself in the coupled GNSS/DORIS configuration. 


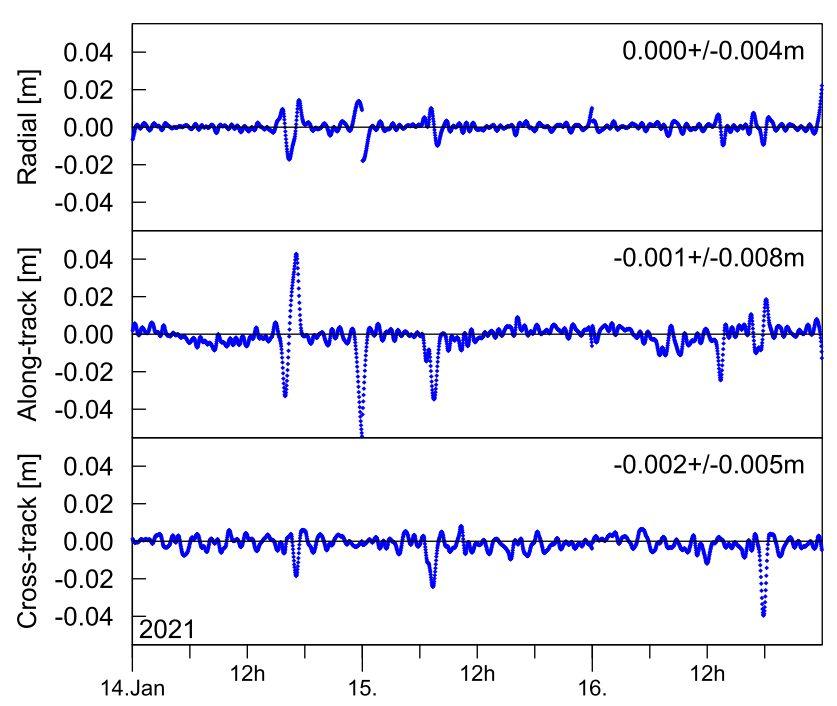

Fig. 5 Comparison of reduced-dynamic orbits based on CNES/CLS products with those based on CODE products for a sample 3-day arc

\subsection{Orbit consistency}

For an assessment of the precision, i.e., repeatability, of the orbit determination solutions we assess the consistency of different Sentinel POD solutions. This includes the comparison of results based on different GNSS orbit and clock products, the comparison of single-constellation solutions with combined GPS/Galileo solutions, and, finally, the comparison of kinematic and reduced-dynamic orbits.

For illustration, Fig. 5 shows the difference of reduceddynamic Sentinel-6A orbits based on GPS/Galileo observations and orbit and clock products from CNES/CLS with those based on CODE products for a representative threeday interval. 3D RMS position difference over this interval amount to $1 \mathrm{~cm}$. The errors are dominated by repeated peaks with typical duration of about one hour and amplitudes at the $5 \mathrm{~cm}$ level, which can be attributed to inconsistent ambiguity fixing for individual passes in the two solutions. Long-term statistics over the a 6-month test period show a matching 3D RMS error and confirm the overall error distribution (Table 8). Overall, 3D RMS differences between the two solutions exceed a $1 \mathrm{~cm}$ threshold in $4.3 \%$ and a $2 \mathrm{~cm}$ threshold in $1.2 \%$ of all epochs. These values appear generally compatible with the ambiguity fixing rates reported in Table 7, but further investigations may be required to obtain an optimum trade-off between fixing rates and the occurrence of false ambiguity fixes.

The summary statistics Table 8 also show a good consistency of reduced-dynamic orbit solutions for the individual constellations with the dual-constellation processing. GPSonly and Galileo-only results are essentially unbiased and only show a slightly larger scatter, which may be related to the lower number of processed satellites and observations.

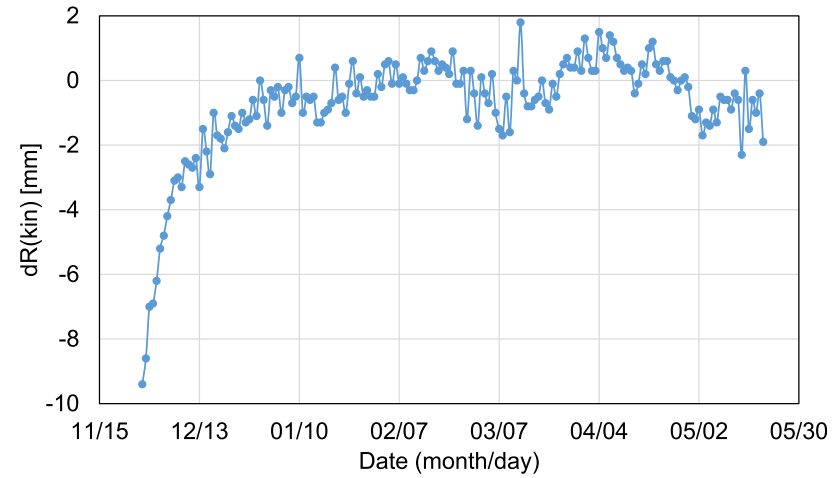

Fig. 6 Variation of radial offset between kinematic and reduceddynamic Sentinel-6A orbits over the first 6 months of the Sentinel-6A mission

Kinematic solutions obtained with previously fixed carrier phase ambiguities from the reduced-dynamic processing exhibit a $1.3 \mathrm{~cm}$ (3D RMS) consistency with the CODEbased reference solution. Errors are most pronounced in the radial direction which simply reflects the higher dilution of precision (DOP) in vertical direction as compared to the horizontal DOP (Langley et al. 2017). Closer inspection of the temporal evolution shows that the radial difference of kinematic and reduced-dynamic positions reveals an almost exponential decrease by roughly $10 \mathrm{~mm}$ over the first month of the Sentinel-6A mission before converging to a stable value near the start of 2021 (Fig. 6).

In the reduced-dynamic POD, the estimated CoM trajectory is constrained in radial direction by the dynamical orbit model. The kinematic POD, in contrast yields an estimate of the antenna position from which the CoM location is obtained based on the assumed CoM offset of the antenna in the spacecraft frame. Varying differences between kinematic and reduced-dynamic orbits might be understood in terms of changes in the CoM location within the spacecraft that are not reflected by the manufacturer's model for CoM shifts in response to fuel consumption and varying tank loads. Alternatively, a mismodeling of the radial acceleration may be considered, in which case the observed differences could be explained (see Eqn. (1)) by an exponentially decreasing radial acceleration with an initial value of about $-25 \mathrm{~nm} / \mathrm{s}^{2}$ that is not considered in the POD process. Given the inbound orientation of the empirical acceleration and its variation in time, outgassing of the solar cells appears as a plausible cause of the observed phenomenon.

\subsection{Empirical accelerations}

As discussed in Sect. 4, the reduced-dynamic approach for Sentinel-6A POD makes use of empirical accelerations in addition to an a priori force model. The magnitude of these accelerations provides an indicator for the quality of the 
Table 8 Performance of reduced-dynamic and kinematic Sentinel-6A orbit determination solutions relative to a reduced-dynamic POD using GPS and Galileo observations and CODE GNSS products. For each axis, the mean \pm standard deviation over a 6-month period are provided. All values in [mm]

\begin{tabular}{|c|c|c|c|c|c|}
\hline Type & GNSS & GNSS product & Radial & Along-track & Cross-track \\
\hline Red. dyn. & GPS+Galileo & CNES/CLS & $-0.1 \pm 2.4$ & $+0.2 \pm 4.5$ & $-0.7 \pm 3.2$ \\
\hline Red. dyn. & GPS & CODE & $+0.1 \pm 3.7$ & $+0.4 \pm 9.0$ & $-0.1 \pm 3.5$ \\
\hline Red. dyn. & Galileo & CODE & $+0.1 \pm 3.1$ & $+0.0 \pm 6.9$ & $-0.3 \pm 3.1$ \\
\hline Kinematic & GPS+Galileo & CNES/CLS & $-1.0 \pm 10.3$ & $+0.3 \pm 6.0$ & $-0.4 \pm 4.6$ \\
\hline Kinematic & GPS+Galileo & CODE & $-0.7 \pm 9.8$ & $+0.1 \pm 3.6$ & $+0.3 \pm 3.2$ \\
\hline
\end{tabular}

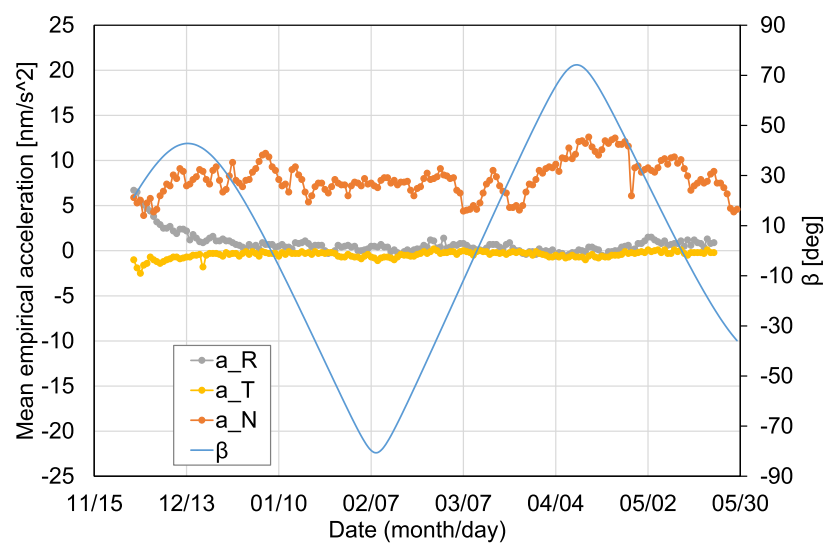

Fig. 7 Daily mean values of empirical acceleration in radial (R), alongtrack $(\mathrm{T})$, and cross-track $(\mathrm{N})$ direction

macro-model for the non-gravitational forces and can also hint at possible errors in the adopted antenna offsets from the center of mass.

Daily mean values of the estimated empirical accelerations in radial, along-track, and cross-track direction are shown in Fig. 7. Comparison with the Sun's elevation $\beta$ above the orbital plane shows essentially no seasonal variation and thus confirms that the macro-model of Table 5 is able to capture the overall variation of solar radiation pressure under a wide range of Sun-orbit geometries and incidence angles.

During the first weeks after launch, gradual variations of the mean accelerations may be observed in all three axes before settling at near-constant values. As discussed above, these variations may be understood by the assumption of outgassing of satellite surfaces during the early orbit phase. Following the initial convergence, near-zero mean values are obtained for accelerations in the radial and along-track direction, whereas a systematic acceleration bias of about $8 \mathrm{~nm} / \mathrm{s}^{2}$ may be recognized in cross-track direction. Following Montenbruck et al. (2018), a constant cross-track acceleration $\Delta a_{\mathrm{N}}$ causes a lateral shift

$$
\Delta r_{\mathrm{N}}=\left(\frac{T}{2 \pi}\right)^{2} \Delta a_{\mathrm{N}}
$$

of the orbital plane and may be required to align the modeled center-of-mass trajectory with the observed GNSS antenna, if the assumed GNSS antenna offset from the center-of-mass does not match the actual value. In case of Sentinel-6A, the estimated cross-track acceleration suggests that the assumed antenna reference point offset $y_{\mathrm{ARP}}-y_{\mathrm{CoM}}=8 \mathrm{~mm}$ (Table 4) is too large by roughly $8 \mathrm{~mm}$.

\subsection{Satellite laser ranging}

Satellite laser ranging constitutes a key technique for independent validation of the GNSS-based POD results of LEO satellites (Arnold et al. 2019). Since the early mission phase, Sentinel-6A has been regularly tracked by stations of the International Laser Ranging Service (ILRS; Pearlman et al. 2019). For the present analysis, a set of 13 high-performance ILRS stations (Arequipa, Graz, Greenbelt, Haleakala, Hartebeesthoek, Herstmonceaux, Matera, Monument Peak, Mount Stromlo, Papete, Potsdam, Yarragadee, and Zimmerwald) has been selected, which offer normal points with precision at the $5-10 \mathrm{~mm}$ level. The modeled station positions are based on the SLRF2014 reference frame and take into account timedependent variations as summarized in Table 4.

The Sentinel-6A LRA is made up of a central prism surrounded by a ring of eight prisms with a $50^{\circ}$ tilt of their viewing directions. The centers of the prisms' front surfaces are located on a common sphere of $82.55 \mathrm{~mm}$ around the optical reference point. For a group refractive index of 1.4853 at $532 \mathrm{~nm}$ and a prism vertex height of $22.5 \mathrm{~mm}$, a range correction of about $49 \mathrm{~mm}$ is obtained for rays perpendicular to any of the prism surfaces. Range corrections for arbitrary incidence angles have been computed for this study based on the LRA design data using a nearest-prism approximation (Montenbruck and Neubert 2011), and are illustrated in Fig. 8.

Residuals of roughly 37000 normal points collected up to end of May 2021 by the 13 ILRS stations are shown in Fig. 9. Compared to the ambiguity-fixed reduced-dynamic orbits obtained with CODE GNSS products, the SLR observations exhibit differences with a mean value of $1.0 \mathrm{~mm}$ and 


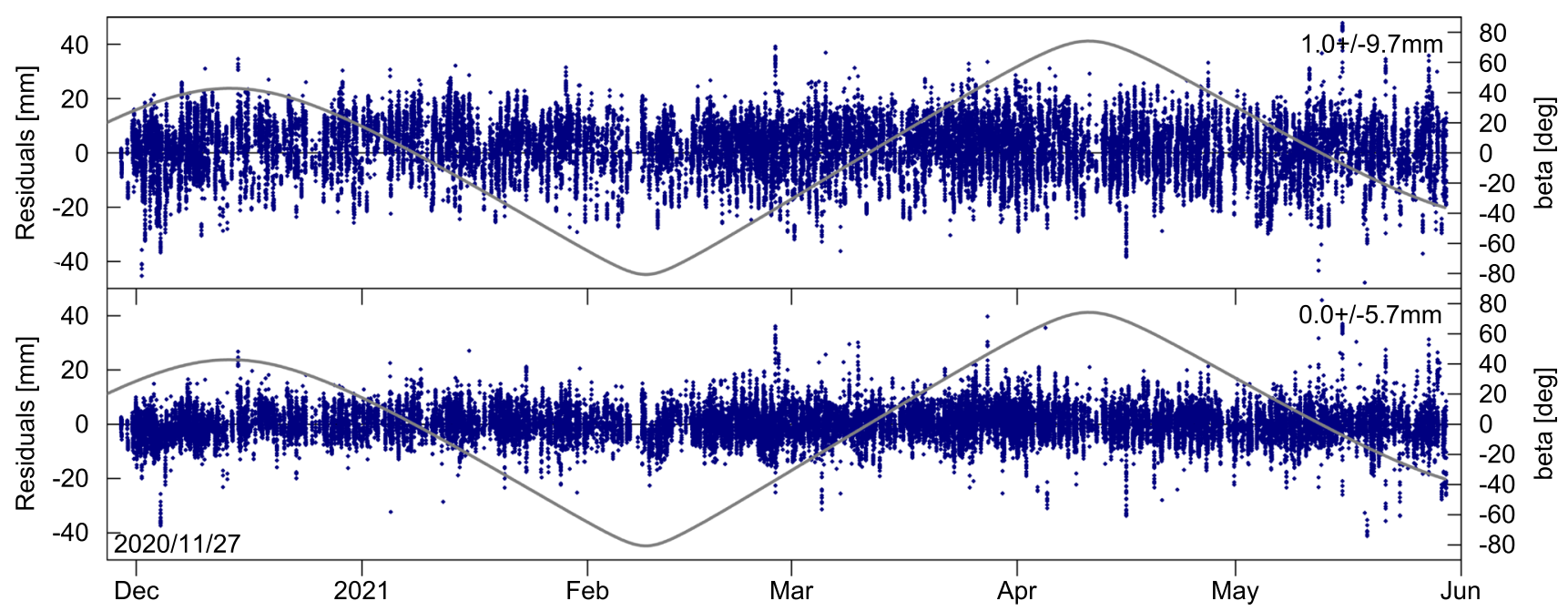

Fig. 9 Sentinal-6A satellite laser ranging residuals before (top) and after (bottom) adjustment of orbit and station offsets. The solid gray lines show the variation of the Sun's angle above the orbit plane ( $\beta$-angle)

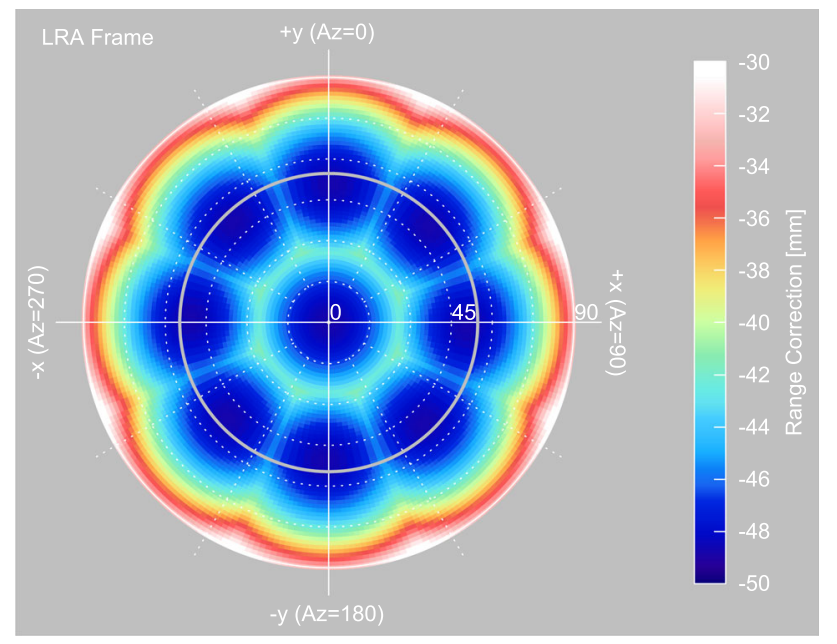

Fig. 8 Range correction of Sentinel-6 LRA relative to the optical reference point. The bold line at $55^{\circ}$ off-boresight angle marks the LRA field of view for stations tracking the Sentinel-A spacecraft above an elevation of $10^{\circ}$

a standard deviation of $9.7 \mathrm{~mm}$. Virtually identical results are obtained with the CNES/CLS-based POD solution.

While these statistics already demonstrate a high overall performance of the Sentinel-6 orbit determination and compatibility with the specification of a better than $1.5 \mathrm{~cm}$ radial error, they are still affected by systematic, cm-level errors in the SLR site coordinates and range biases that mask the actual orbit quality. For calibrating these biases, we made complementary use of ambiguity-fixed orbits of five additional LEO satellites (Swarm-A/B/C, Sentinel-3A/B) based on the same processing standards and CODE GNSS products as the Sentinel-6A POD solutions. Given the large number of observations with good hemispherical coverage of the individual stations, the offsets can be determined in a least-squares adjustment (Arnold et al. 2019) with good confidence. In addition, systematic offsets of the tracked LEO satellite in radial, along-track, and cross-track direction may be adjusted that can be caused by errors in LRA positions, GNSS antenna coordinates, or CoM location. For jointly estimating both station-related corrections and orbit errors, we applied a zero-mean constraint for both the range biases and the up-component of site positions over all contributing stations. Overall, the adjustment almost halves the residuals (Fig. 9) and yields a standard deviation of $5.7 \mathrm{~mm}$ which characterizes the actual precision of the SLR normal points and the GNSS-based POD solutions. Aside from the availability of normal points of individual stations, no obvious seasonal variations can be recognized in the S6A SLR residuals. In particular, no $\beta$-angle dependence is discernible, which might hint at systematic deficiencies in the solar radiation pressure modeling.

From the SLR residuals analysis, mean orbit errors at the few-mm level in radial, along-track, and cross-track direction, or, equivalently, the $-z,+x$, and $-y$ body axis can be inferred for the Sentinel-6A POD solutions (Table 9). Better than $1 \mathrm{~mm}$ consistency of SLR observations and GNSS-based orbit determination is obtained for the radial orbit component relative to the height system defined by the 13 ILRS stations. Aside from the specific choice of stations, this favorable result depends also on the validity of the employed non-gravitational force models, as well as the proper knowledge of CoM location, LRA mount point, and LRA range corrections. Uncertainties in these factors are likely to limit the actual orbit height accuracy to a level of about $5 \mathrm{~mm}$, but further monitoring and comparison of independent POD solutions will be required to verify and consolidate this error budget. 
Table 9 Systematic errors of GNSS-based Sentinel-6A orbits as inferred from SLR observations over a 3-month period

\begin{tabular}{llll}
\hline Component & $\mathrm{R}(-z)$ & $\mathrm{T}(+x)$ & $\mathrm{N}(-y)$ \\
\hline Offset & -0.3 & +2.9 & -5.7
\end{tabular}

All values in $[\mathrm{mm}]$

Concerning the along-track component, multiple causes of the observed $2.9 \mathrm{~mm}$ difference between the GNSS-based orbit determination and the SLR observations can be imagined. These include GNSS antenna phase center offsets or CoM model errors in the longitudinal spacecraft axis but also biases in the timing of GNSS carrier phase observations (Montenbruck et al. 2006), which are essentially unobservable in the POD for the specific S6A attitude. SLR timing biases can likewise contribute to the apparent SLR-GNSS difference. Following Arnold et al. (2019), systematic timing errors at the sub-microsecond level are present in various ILRS stations, which might then map into an apparent alongtrack error at the few-mm level. However, no unambiguous explanation can be given at this stage without independent calibration of the various error sources.

The most pronounced mismatch arises in the cross-track direction (or $-y$ body axis), where the SLR analysis suggests a systematic $-6 \mathrm{~mm}$ error in the LRA trajectory as described by the GNSS-based POD solution. This offset is likewise seen in the ambiguity-fixed kinematic and reduced-dynamic solutions, which eliminates the modeled CoM location within the spacecraft body as a potential error source. Instead, the observed offset indicates that the true separation of the LRA and the GNSS antenna in $y$-direction is about $6 \mathrm{~mm}$ smaller than modeled. While no evidence for such a modeling error is available from the manufacturer documentation, it is instructive to note that a similar ( $8 \mathrm{~mm}$; see Sect. 5.4) discrepancy can be inferred for the CoM offset of the GNSS antenna offset from the analysis of empirical accelerations estimated in the POD process. Overall, both SLR residuals and empirical accelerations can thus be explained by assuming a modified GNSS antenna reference point location $y_{\text {ARP }}$ of about -6 to $-8 \mathrm{~mm}$ instead of the nominal position $y_{\mathrm{ARP}}=0 \mathrm{~mm}$.

\section{Summary and conclusions}

Sentinel-6A is the first science mission in low Earth orbit using a dual-constellation, GPS-Galileo receiver for precise orbit determination. Based on data from the first 6 months of the Sentinel-6A mission, the achievable orbit precision and accuracy were assessed. Compared to GPS tracking, Galileo offers reduced measurement errors and yields an improved success rate in single-receiver ambiguity fixing. On the other hand, the Galileo constellation still offers a notably lower number of operational satellites placed in only three rather than six orbital planes. Despite these differences, reduced-dynamic POD solutions using only GPS or Galileo observations exhibit very good (better than $1 \mathrm{~cm} 3 \mathrm{D}$ RMS) consistency with the solutions based on a combined set of GPS and Galileo observations.

Processing of undifferenced GPS and Galileo observations with ambiguity fixing is presently supported by precise GNSS orbit clock, and bias products of two IGS analysis centers (CNES/CLS and CODE), which are made available on a weekly basis with 4-7 days latency. POD results obtained with the two types of GNSS products show 3D RMS differences of well below $1 \mathrm{~cm}$ over the entire data period. However, peak errors at the $5 \mathrm{~cm}$ level can be noted over representative intervals of $0.5-1 \mathrm{~h}$ that are attributed to erroneously fixed ambiguities for individual passes in one of the two solutions. Further adaptation of acceptance criteria for the wide-/narrow-lane ambiguity fixing may be required to reduce the occurrence of such false fixes at the possible expense of a reduced overall fixing rate.

For a consistent processing of all measurements, various calibrations of the Sentinel-6A GNSS receiver and antenna are required. These include a pre-mission or inflight calibration of hardware related inter-signal code and phase biases between semi-codeless tracking of the $\mathrm{P}(\mathrm{Y})$ signals and tracking of the civil L2C signal of GPS as a prerequisite for ambiguity fixing with mixed signal sets. Secondly, proper antenna phase center calibration in the frequency bands used for GPS (L1/L2) and Galileo (E1/E5a) tracking is required. In view of obvious $(\Delta z \approx 25 \mathrm{~mm})$ discrepancies between manufacturer-specified and observed phase center locations relative to the spacecraft center-of-mass, an inflight calibration is indispensable for proper modeling of carrier phase observations in the POD process. Unfortunately, GNSS can no longer provide independent height information after adjustment of the antenna coordinates, and the orbital radius determined in the POD process is ultimately tied to the dynamic orbit models. Errors in these models are mainly related to the non-gravitational forces with presumed uncertainties at the 10-20\% level. In radial direction, accelerations due to solar and Earth radiation pressure as predicted by the current macro-model exhibit representative values of $50 \mathrm{~nm} / \mathrm{s}^{2}$ and $35 \mathrm{~nm} / \mathrm{s}^{2}$, respectively. This translates into a $9-17 \mathrm{~nm} / \mathrm{s}^{2}$ uncertainty of the radial acceleration and finally a 3-7 mm uncertainty in the height of the resulting center-ofmass trajectory.

Independent verification of the resulting POD accuracy is achieved through comparison with satellite laser ranging where 1-cm consistency of measured normal points with the GNSS-based orbits is achieved and a mean height error of less than $2 \mathrm{~mm}$ is identified. Following adjustment of stationspecific position corrections and range biases as well as average orbit/sensor offsets, a $6 \mathrm{~mm}$ standard deviation of 
the SLR normal point residuals is achieved, which further evidences an excellent precision of the POD results.

Acknowledgements Sentinel-6A flight and pre-mission test data used in this study have kindly been made available by EUMETSAT and the European Commission (EC) as part of the Copernicus program. We also appreciate the continued technical discussion with the European Space Agency (ESA), the Jet Propulsion Laboratory as well as the Quality Working Group of the Copernicus Precise Orbit Determination Service (CPOD). Our study builds extensively on GNSS orbit, clock, and bias products facilitating ambiguity resolution, which are made available by the CNES/CLS (Centre National d'Études Spatiales / Collecte Localisation Satellites) analysis center and Center for Orbit Determination in Europe (CODE) of the International GNSS Service (IGS). Provision of this community service is greatly appreciated and acknowledged. The authors are, furthermore, grateful to the International Laser Ranging Service (ILRS) for their continued effort to collect and publicly provide the SLR observations of Sentinel-6A and other LEO satellites. We would also like to acknowledge valuable discussions on the choice of a tailored Sentinel-6A macro-model with H. Peter and F. Gini.

Author Contributions OM proposed the idea of the contribution, designed the experiments, analyzed the results and wrote the paper. $\mathrm{SH}$ and MW contributed to the GNSS data processing and SLR analysis. FZ contributed to the instrument calibration and technical understanding. $\mathrm{SH}, \mathrm{MW}$, and FZ reviewed the final manuscript.

Funding Open Access funding enabled and organized by Projekt DEAL.

Data availibility GNSS and SLR data used are publicly available from International GNSS Service and International Satellite Laser Ranging Service from the respective data centers.

Open Access This article is licensed under a Creative Commons Attribution 4.0 International License, which permits use, sharing, adaptation, distribution and reproduction in any medium or format, as long as you give appropriate credit to the original author(s) and the source, provide a link to the Creative Commons licence, and indicate if changes were made. The images or other third party material in this article are included in the article's Creative Commons licence, unless indicated otherwise in a credit line to the material. If material is not included in the article's Creative Commons licence and your intended use is not permitted by statutory regulation or exceeds the permitted use, you will need to obtain permission directly from the copyright holder. To view a copy of this licence, visit http://creativecomm ons.org/licenses/by/4.0/.

\section{References}

Arnold D, Montenbruck O, Hackel S, Sośnica K (2019) Satellite laser ranging to low earth orbiters: orbit and network validation. J Geodesy 93(11):2315-2334. https://doi.org/10.1007/s00190018-1140-4

Auriol A, Tourain C (2010) DORIS system: the new age. Adv Space Res 46(12):1484-1496. https://doi.org/10.1016/j.asr.2010.05.015

Banville S, Geng J, Loyer S, Schaer S, Springer T, Strasser S (2020) On the interoperability of IGS products for precise point positioning with ambiguity resolution. J Geodesy 94(1):1-15. https://doi.org/ 10.1007/s00190-019-01335-w

Cerri L, Berthias J, Bertiger W, Haines B, Lemoine F, Mercier F, Ries J, Willis P, Zelensky N, Ziebart M (2010) Precision orbit determination standards for the Jason series of altimeter missions.
Marine Geod 33(S1):379-418. https://doi.org/10.1080/01490419. 2010.488966

Cullen R (2021) Sentinel-6A POD context, JC-TN-ESA-0420, v1.4, 1 Feb. 2021

Cullen R, Francis R (2014) The ocean surface topography JasonCS/Sentinel-6 mission. In: AGU fall meeting abstracts, vol 2014, pp OS33C-1093

Dach R, Schaer S, Arnold D, Kalarus M, Prange L, Stebler P, Villiger A, Jaeggi A (2020) CODE rapid product series for the IGS. https://doi.org/10.7892/boris.75854.4, http://www.aiub.unibe.ch/ download/CODE

Debaisieux A, Aubry JP, Gerard E, Brunet M (1985) A satellite oscillator for very precise orbitography: The DORIS program. In: 39 th annual symposium on frequency control. IEEE, pp 202-211. https://doi.org/10.1109/FREQ.1985.200846

Donlon CJ, Cullen R, Giulicchi L, Vuilleumier P, Francis CR, Kuschnerus M, Simpson W, Bouridah A, Caleno M, Bertoni R et al (2021) The Copernicus Sentinel-6 mission: enhanced continuity of satellite sea level measurements from space. Remote Sens Environ 258(112):395. https://doi.org/10.1016/j.rse.2021.112395

Doornbos E (2012) Thermospheric density and wind determination from satellite dynamics. Springer, Heidelberg

Fernández J, Peter H, Calero EJ, Berzosa J, Gallardo LJ, Féménias P (2019) Sentinel-3A: validation of orbit products at the Copernicus POD service. In: Fiducial reference measurements for altimetry. Springer, pp 75-82. https://doi.org/10.1007/1345_2019_64

Gonzalez F, Waller P (2007) Short term GNSS clock characterization using one-way carrier phase. In: IEEE international frequency control symposium joint with the 21st European frequency and time forum. IEEE, pp 517-522. https://doi.org/10.1109/FREQ.2007. 4319127

Hatch R (1983) The synergism of GPS code and carrier measurements. In: International geodetic symposium on satellite Doppler positioning 2:1213-1231

Hess WN (1968) The radiation belt and magnetosphere. Waltham

Huang W, Männel B, Brack A, Schuh H (2021) Two methods to determine scale-independent GPS PCOs and GNSS-based terrestrial scale: comparison and cross-check. GPS Solut 25(1):1-13. https:// doi.org/10.1007/s10291-020-01035-5

Jäggi A, Dach R, Montenbruck O, Hugentobler U, Bock H, Beutler G (2009) Phase center modeling for LEO GPS receiver antennas and its impact on precise orbit determination. J Geodesy 83(12):11451162. https://doi.org/10.1007/s00190-009-0333-2

Jalabert E, Mercier F (2018) Analysis of South Atlantic Anomaly perturbations on Sentinel-3A ultra stable oscillator. Impact on DORIS phase measurement and DORIS station positioning. Adv Space Res 62(1):174-190

Jayles C, Exertier P, Martin N, Chauveau J, Samain E, Tourain C, Auriol A, Guillemot P (2016) Comparison of the frequency estimation of the DORIS/Jason2 oscillator thanks to the onboard DIODE and Time Transfer by Laser Link experiment. Adv Space Res 58(12):2601-2616. https://doi.org/10.1016/j.asr.2015.08.033

Johnston G, Riddell A, Hausler G (2017) The international GNSS service. In: Teunissen $\mathrm{P}$, Montenbruck O (eds) Springer handbook of global navigation satellite systems. Springer, chap 33, pp 967-982. https://doi.org/10.1007/978-3-319-42928-1_33

Katsigianni G, Loyer S, Perosanz F, Mercier F, Zajdel R, Sośnica K (2019) Improving Galileo orbit determination using zerodifference ambiguity fixing in a multi-GNSS processing. Adv Space Res 63(9):2952-2963

Knocke PC, Ries JC, Tapley BD (1988) Earth radiation pressure effects on satellites. In: AIAA/AAS astrodynamics conference, pp 577587

Kobel C, Arnold D, Jäggi A (2019) Combination of precise orbit solutions for Sentinel-3A using variance component estimation. Adv Geosci 50:27-37. https://doi.org/10.5194/adgeo-50-27-2019 
Kouba J (2019) Relativity effects of Galileo passive hydrogen maser satellite clocks. GPS Solut 23(4):1-11. https://doi.org/10.1007/ s10291-019-0910-7

Langley RB, Teunissen PJG, Montenbruck O (2017) Introduction to GNSS. In: Teunissen P, Montenbruck O (eds) Springer handbook of global navigation satellite systems. Springer, chap 1, pp 3-23. https://doi.org/10.1007/978-3-319-42928-1_1

Laurichesse D, Mercier F, Berthias JP, Broca P, Cerri L (2009) Integer ambiguity resolution on undifferenced GPS phase measurements and its application to PPP and satellite precise orbit determination. Navigation 56(2):135-149. https://doi.org/10.1002/j.2161-4296. 2009.tb01750.x

Loyer S, Perosanz F, Mercier F, Capdeville H, Marty JC (2012) Zerodifference GPS ambiguity resolution at CNES-CLS IGS analysis center. J Geodesy 86(11):991. https://doi.org/10.1007/s00190012-0559-2

Lyard F, Lefevre F, Letellier T, Francis O (2006) Modelling the global ocean tides: modern insights from FES2004. Ocean Dyn 56(56):394-415. https://doi.org/10.1007/s10236-006-0086-X

Maiwald F, Brown ST, Koch T, Milligan L, Kangaslahti P, Schlecht E, Skalare A, Bloom M, Torossian V, Kanis J et al (2020) Completion of the AMR-C instrument for Sentinel-6. IEEE J Sel Top Appl Earth Observ Remote Sens 13:1811-1818. https://doi.org/ 10.1109/JSTARS.2020.2991175

Mayer-Gürr T, Pail R, Schuh WD, Kusche J, Baur O, Jäggi A (2012) The new combined satellite only model GOCO03s. In: International symposium on gravity, geoid and height systems, Venice, Italy

Melbourne W (1985) The case for ranging in GPS based geodetic systems. In: Goad C (ed) Proceedings of 1st international symposium on precise positioning with the global positioning system, NOAA, pp 373-386

Mendes V, Pavlis E (2004) High-accuracy zenith delay prediction at optical wavelengths. Geophys Res Lett 31(14):L14,602. https:// doi.org/10.1029/2004GL020308

Milani A, Nobili AM, Farinella P (1987) Non-gravitational perturbations and satellite geodesy. Adam Hilger, Bristol

Montenbruck O, Neubert R (2011) Range correction for the CryoSat and GOCE laser retroreflector arrays, DLR/GSOC TN 11-01. https:// ilrs.cddis.eosdis.nasa.gov/docs/TN_1101_IPIE_LRA_v1.0.pdf

Montenbruck O, Van Helleputte T, Kroes R, Gill E (2005) Reduced dynamic orbit determination using GPS code and carrier measurements. Aerosp Sci Technol 9(3):261-271. https://doi.org/10.1016/ j.ast.2005.01.003

Montenbruck O, Garcia-Fernandez M, Williams J (2006) Performance comparison of semicodeless GPS receivers for LEO satellites. GPS Solut 10(4):249-261. https://doi.org/10.1007/s10291-006-00259

Montenbruck O, Andres Y, Bock H, van Helleputte T, van den Ijssel J, Loiselet M, Marquardt C, Silvestrin P, Visser P, Yoon Y (2008) Tracking and orbit determination performance of the GRAS instrument on MetOp-A. GPS Solut 12(4):289-299. https://doi.org/10. 1007/s10291-008-0091-2

Montenbruck O, Garcia-Fernandez M, Yoon Y, Schön S, Jäggi A (2009) Antenna phase center calibration for precise positioning of LEO satellites. GPS Solut 13(1):23. https://doi.org/10.1007/s10291008-0094-z

Montenbruck O, Hackel S, Jäggi A (2018) Precise orbit determination of the Sentinel-3A altimetry satellite using ambiguity-fixed GPS carrier phase observations. J Geodesy 92(7):711-726. https://doi. org/10.1007/s00190-017-1090-2

Öhgren M, Bonnedal M, Ingvarson P (2011) GNSS antenna for precise orbit determination including s/c interference predictions. In: Proceedings of 5th European conference on antennas and propagation (EUCAP), pp 1990-1994

Pearlman MR, Noll CE, Pavlis EC, Lemoine FG, Combrink L, Degnan JJ, Kirchner G, Schreiber U (2019) The ILRS: approaching 20 years and planning for the future. J Geodesy 93(11):2161-2180. https://doi.org/10.1007/s00190-019-01241-1

Picone JM, Hedin AE, Drob DP, Aikin AC (2002) NRLMSISE-00 empirical model of the atmosphere: statistical comparisons and scientific issues. J Geophys Res 107(A12). https://doi.org/10.1029/ 2002JA009430

Prange L, Villiger A, Sidorov D, Schaer S, Beutler G, Dach R, Jäggi A (2020) Overview of CODE's MGEX solution with the focus on galileo. Adv Space Res 66(12):2786-2798. https://doi.org/10. 1016/j.asr.2020.04.038

Priestley KJ, Smith GL, Thomas S, Cooper D, Lee RB, Walikainen D, Hess P, Szewczyk ZP, Wilson R (2011) Radiometric performance of the CERES Earth radiation budget climate record sensors on the EOS Aqua and Terra spacecraft through April 2007. J Atmos Ocean Tech 28(1):3-21. https://doi.org/10.1175/ 2010JTECHA1521.1

Ray R (1999) A global ocean tide model from TOPEX/POSEIDON altimetry: GOT99.2, NASA technical memorandum 209478

Rebischung P (2020) Switch to IGb14 reference frame, IGSMAIL7921, 14 April 2020. https://lists.igs.org/pipermail/igsmail/2020/ 007917.html

Rebischung P, Schmid R (2016) IGS14/igs14.atx: a new framework for the IGS products. In: AGU Fall Meeting, pp G41A-0998

Romero I (2020) RINEX - the receiver independent exchange format, v. 3.05, 1 December 2020

Roselló J, Silvestrin P, Weigand R, d'Addio S, Rodríguez AG, Risueño GL (2012) Next generation of ESA's GNSS receivers for Earth observation satellites. In: 2012 6th ESA workshop on satellite navigation technologies (Navitec 2012) \& European workshop on GNSS signals and signal processing. IEEE, pp 1-8

Schaer S, Villiger A, Arnold D, Dach R, Prange L, Jäggi A (2021) The CODE ambiguity-fixed clock and phase bias analysis products: generation, properties, and performance. J Geodesy 95(7):1-25. https://doi.org/10.1007/s00190-021-01521-9

Scharroo R, Bonekamp H, Ponsard C, Parisot F, von Engeln A, Tahtadjiev M, de Vriendt K, Montagner F (2016) Jason continuity of services: continuing the Jason altimeter data records as Copernicus Sentinel-6. Ocean Sci 12(2):471-479. https://doi.org/10.5194/os12-471-2016

Shampine LF, Gordon MK (1975) Computer solution of ordinary differential equations: the initial value problem. W. H. Freeman, San Francisco

Štěpánek P, Duan B, Filler V, Hugentobler U (2020) Inclusion of GPS clock estimates for satellites Sentinel-3A/3B in DORIS geodetic solutions. J Geodesy 94(12):1-20. https://doi.org/10.1007/ s00190-020-01428-x

Sutton EK (2009) Normalized force coefficients for satellites with elongated shapes. J Spacecr Rocket 46(1):112-116. https://doi.org/10. 2514/1.40940

van den IJssel J, Forte B, Montenbruck O (2016) Impact of Swarm GPS receiver updates on POD performance. Earth Planets Space 68(1):1-17. https://doi.org/10.1186/s40623-016-0459-4

Villiger A, Schaer S, Dach R, Prange L, Sušnik A, Jäggi A (2019) Determination of GNSS pseudo-absolute code biases and their long-term combination. J Geodesy 93(9):1487-1500

Villiger A, Dach R, Schaer S, Prange L, Zimmermann F, Kuhlmann H, Wübbena G, Schmitz M, Beutler G, Jäggi A (2020) GNSS scale determination using calibrated receiver and Galileo satellite antenna patterns. J Geodesy 94(9):1-13. https://doi.org/10.1007/ s00190-020-01417-0

VMF (2021) VMF data server. https://doi.org/10.17616/R3RD2H

Wang N, Yuan Y, Li Z, Montenbruck O, Tan B (2016) Determination of differential code biases with multi-GNSS observations. J Geodesy 90(3):209-228. https://doi.org/10.1007/s00190-015-0867-4

Wijaya DD, Böhm J, Karbon M, Kràsnà H, Schuh H (2013) Atmospheric pressure loading. In: Atmospheric effects in space geodesy. 
Springer, pp 137-157. https://doi.org/10.1007/978-3-642-369322_4

Wu SC, Yunck TP, Thornton CL (1991) Reduced-dynamic technique for precise orbit determination of low earth satellites. J Guid Control Dyn 14(1):24-30

Wu JT, Wu SC, Hajj G, Bertiger WI, Lichten SM (1993) Effects of antenna orientation on GPS carrier phase. Manuscr Geodaet 18(2):91-98
Wübbena G (1985) Software developments for geodetic positioning with GPS using TI 4100 code and carrier measurements. In: Goad $\mathrm{C}$ (ed) Proceedings of 1 st international symposium on precise positioning with the global positioning system, NOAA, pp 403-412

Young L (2017) JPL GNSS receivers, past, present, and future. In: SCaN/HEOMD workshop on emerging technologies for autonomous space navigation, NASA 